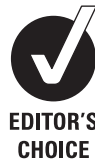

\title{
Prevention of sport injury Il: a systematic review of clinical science research
}

\author{
Kellen McBain, ${ }^{1}$ lan Shrier, ${ }^{2,3}$ Rebecca Shultz, ${ }^{3,4}$ Willem H Meeuwisse, ${ }^{3,5}$ Martin Klügl, \\ Daniel Garza, ${ }^{1}$ Gordon 0 Matheson ${ }^{1,3}$
}

\begin{abstract}
- Additional appendix 1 is published online. To view this file please visit the journal online (http://bjsm.bmj.com/ content/46/3.toc).

'Division of Sports Medicine, Department of Orthopaedic

Surgery, Stanford University School of Medicine, Palo Alto, California, USA

${ }^{2}$ Centre for Clinical Epidemiology and Community Studies, Lady Davis Institute for Medical Research, Jewish General Hospital, McGill University, Montreal, Quebec, Canada

${ }^{3}$ RAISEM: Research Alliance in Sport and Exercise Medicine ${ }^{4}$ Human Performance Laboratory, Department of Orthopaedic Surgery, Stanford University School of Medicine, Palo Alto, California, USA ${ }^{5}$ Sport Injury Prevention Research Centre, University of Calgary, Calgary, Alberta, Canada
\end{abstract}

\section{Correspondence to} lan Shrier, Centre for Clinical Epidemiology and Community Studies, Lady Davis Institute for Medical Research, Jewish General Hospital, 3755 Cote Ste-Catherine Road, Montreal OC H2T 2Y6, Canada; ian.shrier@mcgill.ca

Received 29 October 2010 Accepted 2 March 2011 Published Online First 6 April 2011

\author{
ABSTRACT \\ Objective To characterise the nature of the sport injury \\ prevention literature by reviewing published articles \\ that evaluate specific clinical interventions designed to \\ reduce sport injury risks.
}

Data sources PubMed, Cinahl, Web of Science and Embase.

Main results Only 139 of 2525 articles retrieved met the inclusion criteria. Almost $40 \%$ were randomised controlled trials and $30.2 \%$ were cohort studies.

The focus of the study was protective equipment in $41 \%$, training in $32.4 \%$, education in $7.9 \%$, rules and regulations in $4.3 \%$, and $13.3 \%$ involved a combination of the above. Equipment research studied stability devices $(42.1 \%)$, head and face protectors (33.3\%), attenuating devices (17.5\%) as well as other devices (7\%). Training studies often used a combination of interventions (eg, balance and stretching); most included balance and coordination (63.3\%), with strength and power (36.7\%) and stretching (22.5\%) being less common. Almost $70 \%$ of the studies examined lower extremity injuries, and a majority of these were joint (non-bone)-ligament injuries. Contact sports were most frequently studied (41.5\%), followed by collision (39.8\%) and non-contact (20.3\%).

Conclusion The authors found only 139 publications in the existing literature that examined interventions designed to prevent sports injury. Of these, the majority investigated equipment or training interventions whereas only $4 \%$ focused on changes to the rules and regulations that govern sport. The focus of intervention research is on acute injuries in collision and contact sports whereas only $20 \%$ of the studies focused on non-contact sports.

\section{INTRODUCTION}

Although physical activity is associated with an improved quality of $\operatorname{life}^{12}$ and an overall reduction in mortality ${ }^{3}$ and morbidity, ${ }^{4}$ there remains an associated activity-related risk of injury and re-injury. ${ }^{6} 7$ Injury prevention research plays an important role in the promotion of safe exercise participation by identifying risk factors for injury and re-injury. Indeed, global interest in injury prevention research has increased over the past decade with two previous World Congress on Sport Injury Prevention occurring and a third Congress is scheduled for April 2011. Further, Engebretsen and $\mathrm{Bahr}^{8}$ searched PubMed using the keywords 'athletic injury' and 'prevention' and found that the number of clinical trials and randomised controlled trials (RCTs) approximately doubled between 2000 and 2005. In addition, a recent review of published English literature titles and abstracts addressing sport injury prevention $(\sim 12000 \text { articles })^{9}$ found a steady increase in these articles over the past 15-20 years.

Despite the increase in sport injury prevention research, Chalmers has questioned its quality ${ }^{10}$ as less than $50 \%$ of the articles on sport injury prevention were original research and most of the original research examined incidence and aetiology. ${ }^{9}$ Only 492 articles actually evaluated the efficacy or effectiveness of sport injury prevention interventions. ${ }^{9}$

The first step towards improving future prevention programmes is to better understand past and current sport injury prevention interventions. In a companion paper in this issue, we provide an overview of studies that examined the effect of interventions designed to reduce known risk factors for injury (K McBain, I Shrier, R Shultz et al, manuscript submitted). Although these types of studies provide information more rapidly than studies evaluating actual changes in injury rates (because the latter require much larger sample sizes studied over longer periods of time), the ultimate objective of injury prevention research is to actually reduce injuries. The purpose of this paper is to provide an overview of published articles (categorised by study design, intervention, injury location, injury type and sport) that examined the clinical outcomes of a change in injury risk or injury rate.

\section{METHODS}

We searched PubMed, Cinahl, Embase and Web of Science electronic databases using the search strategy in appendix 1 to identify all potentially relevant English language articles. After reviewing titles, abstracts and full text of potentially relevant articles, we excluded any article that was not original research or that did not report either injury risk or injury rate. We also performed a manual search of the bibliographies to identify papers missed during the electronic search. One reviewer extracted all data using a standardised form and a second reviewer assessed the work for errors; discrepancies were resolved by consensus. The data extracted were age and sex of participants, injury history (first-time, recurrent), study design, type of intervention, location of injury, type of injury and sport (see table 1 for detailed category information).

We classified participants' age into three groups: $<18,18-24$ and $\geq 25$ using logical assumptions if the age range was not mentioned (eg, a study with senior players mean (SD) age of 25 (1.5) was 
classified as both $18-24$ and $\geq 25$ ). Concerning injury history, all articles that specifically mentioned recurrence, re-injury or second injury were grouped together as recurrent injury and all others as first-time. Study designs included crossover studies (each subject received all comparison interventions), RCT, pre-post design, observational cohort design, case-series (no comparison group) and cross-sectional design (exposure and outcome measured at the same time). For studies with multiple study designs (because of different analyses), we classified them based on the most important outcome.

Interventions were grouped according to the mechanism of action: (1) 'equipment' if it examined protective devices, (2) 'training' if the objective was to induce a neuromuscular adaptation, (3) 'regulations' if the intervention involved changes in the rules or laws related to sport/activity and (4) 'education' if the programme was designed to improve knowledge that would lead to a reduced injury risk/rate. We subclassified equipment studies as follows: (1.1) stability device, (1.2) attenuating device, (1.3) head and face protectors (included as a separate category because of the number of relevant articles) and (1.4) other. We subclassified training studies as follows: (2.1) balance and coordination, (2.2) strength and power, (2.3) stretching and (2.4) other. In cases where the training programmes could be categorised into more than one group, we used all of the relevant categories (ie, training using a wobble board and plyometric exercises was categorised as both balance and coordination and strength and power).

For injury locations and injury types, we used categories similar to previous publications. ${ }^{11} 12$ In brief, locations included

Table 1 Succinct summary of data abstraction form providing study inclusion and exclusion criteria and categories used during the data abstraction process

\begin{tabular}{|c|c|}
\hline Item & Terms \\
\hline Inclusion criteria & $\begin{array}{l}\text { Language:English } \\
\text { Topic:Injury prevention } \\
\text { Population:Human } \\
\text { Outcome:Injury rates or injury risk }\end{array}$ \\
\hline Age categories* & $<18 ; 18-24 ; \geq 25$ \\
\hline Injury history* & $\begin{array}{l}\text { Recurrent:Specifically mentions recurrence, } \\
\text { re-injury, second injury } \\
\text { First-time:All other articles }\end{array}$ \\
\hline Study design & $\begin{array}{l}\text { Randomised controlled trial; crossover; } \\
\text { pre-post; cohort; case-series, cross-sectional }\end{array}$ \\
\hline Intervention type* & $\begin{array}{l}\text { Equipment:Stability device (eg, athletic tape, shoe } \\
\text { type, wrist guards, ski boots); attenuation device } \\
\text { (eg, shock absorbing insoles, safety balls, impact } \\
\text { bases); head/face protectors; other (eg, playing } \\
\text { surface, thigh protector) } \\
\text { Training:Balance/coordination (eg, wobble board, } \\
\text { balance foam); strength/power (eg, plyometric, } \\
\text { eccentric); stretching (eg, stretching affects on } \\
\text { position sense, reflex activity); other (eg, proper } \\
\text { landing technique, supervised training feedback } \\
\text { instructions, verbal instruction) } \\
\text { Regulation }\end{array}$ \\
\hline Injury location* & $\begin{array}{l}\text { Head/neck/spine; trunk; upper extremity; } \\
\text { lower extremity; other }\end{array}$ \\
\hline Injury type* & $\begin{array}{l}\text { Central-peripheral nervous system (eg, concussion, } \\
\text { nerve, spinal cord compression, brain); } \\
\text { contusion-laceration-abrasion; fracture-bone } \\
\text { stress; joint (non-bone)-ligament (eg, sprains, } \\
\text { cartilage, patello-femoral syndrome); } \\
\text { muscle-tendon; other (eg, soreness) }\end{array}$ \\
\hline Sport type & See appendix 1 \\
\hline
\end{tabular}

\footnotetext{
*Where articles included more than one category, we used all relevant categories.
}

head/neck/spine, upper extremity, trunk lower extremity and other, and injury types included central-peripheral nervous systems, contusion-laceration-abrasion, fracture-bone stress, joint (non-bone)-ligament, muscle-tendon and other.

We extracted each sport mentioned and grouped them for analysis based on the same criteria as our companion paper. In brief, collision/combat sports were grouped together and included alpine skiing/snowboarding because of the high risk of high-speed collision with the surface of the hill. Sports that involved very little risk of contact at all were grouped together (eg, swimming, squash), as were sports where the contact occurs frequently (eg, basketball, baseball). Military studies were excluded from this analysis. If a study examined more than one sport, it was included under each sport and counted more than once.

\section{RESULTS}

The literature search yielded 139 articles considered relevant for analysis. The flow of included and excluded articles is shown in figure 1. Our search yielded 2525 papers, and 330 were considered relevant after the title and abstract search. The bibliography search yielded an additional 71 papers (total $\mathrm{n}=401$ ). Of these, 118 were excluded because they did not meet our inclusion criteria after reading the full text, and 144 articles were excluded because they did not measure clinical outcomes.

\section{Population demographics (age, sex, injury history)}

The age group of participants was defined in 120 articles; $14.2 \%$ only included participants $<18,16.6 \%$ only included participants within the age range $18-24,4.2 \%$ only included participants $\geq 25$ and more than $65 \%$ included participants in

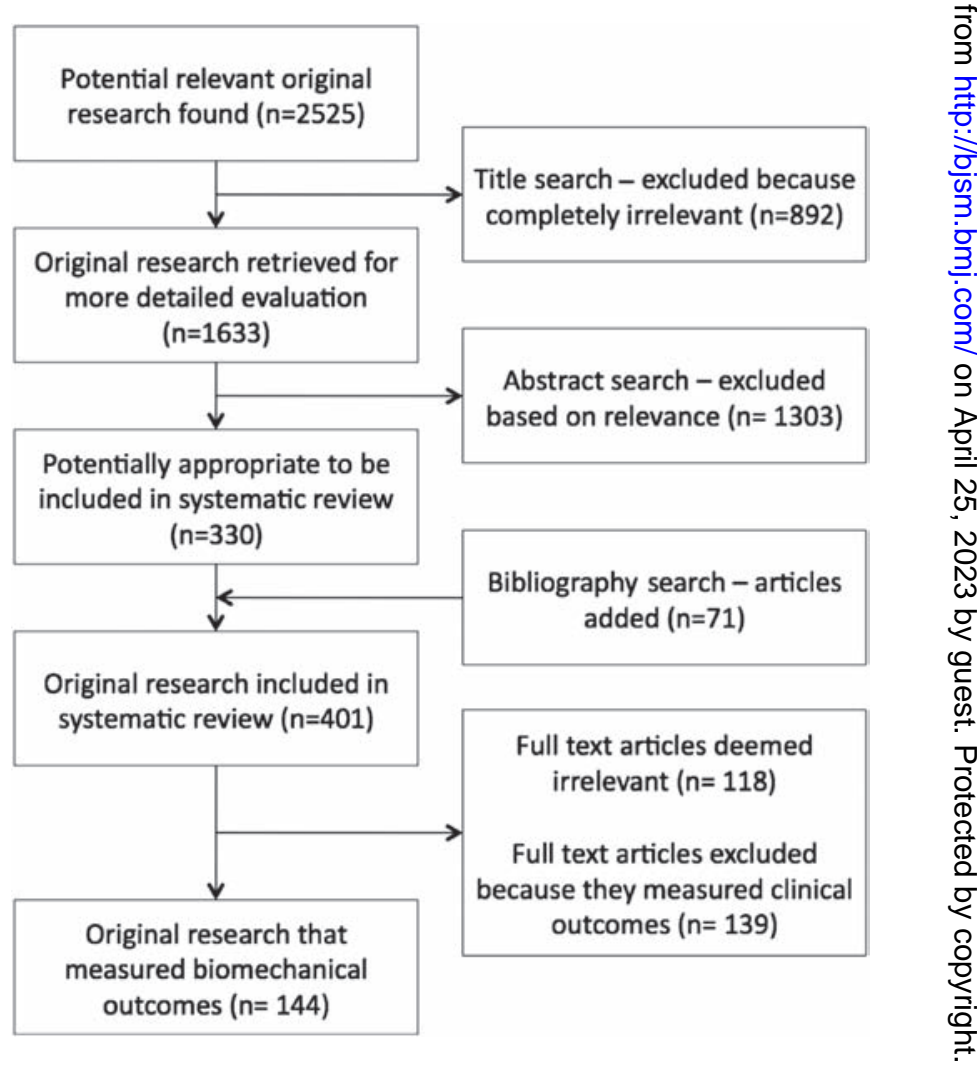

Figure 1 Flow diagram illustrating the number of articles that meet inclusion and exclusion criteria. 
more than one category. Sex was defined in 138 articles; $39.9 \%$ studied exclusively male participants, $13 \%$ studied exclusively female participants and $47.1 \%$ included both sexes. Injury history was clearly cited in 62 articles; $27.4 \%$ focused on preventing first-time injuries, $12.9 \%$ on recurrent injury and $59.7 \%$ included both.

\section{Study design}

The number of articles published over the years stratified by publication type is shown in figure 2. In the years prior to 2006, cohort and RCT study designs were approximately equally preferred. More recently, RCT studies have increased in frequency. Over the entire period, 38.9\% of the clinical injury prevention programmes used a RCT study design, $30.2 \%$ used a cohort design, 18\% used a pre-post design and $12.9 \%$ used either a crossover, cross-sectional, case-series, case-control or ecological study design.

\section{Intervention type}

Figure 3 illustrates the number of injury prevention articles published over time stratified by the type of intervention. Protective equipment was the focus of $41 \%$ of articles $(n=57)$, training $32.4 \%(n=45)$, regulations $4.3 \%(n=6)$ and educational programmes $7.9 \%(n=11)$. The remaining $14.4 \%$ used some combination of intervention strategies.

Figure 4 provides additional detail about the equipment (A) and training (B) studies. Over the entire period, $42.1 \%$ of equipment studies used a stability device, $33.3 \%$ used a head/face protector, $17.5 \%$ used an attenuating device and the remaining 7\% used another type of equipment. Of note, 2001-2005 period included a spike in the number of studies for head/ face protection. Training, balance and coordination interventions increased dramatically since the early 1990s whereas articles related to strength and power began to increase in the early 2000s. More recently, studies investigating stretching have increased. Overall (many studies included multiple components so the total is greater than 100\%), $68.8 \%$ of studies included a balance and coordination component, $36.7 \%$

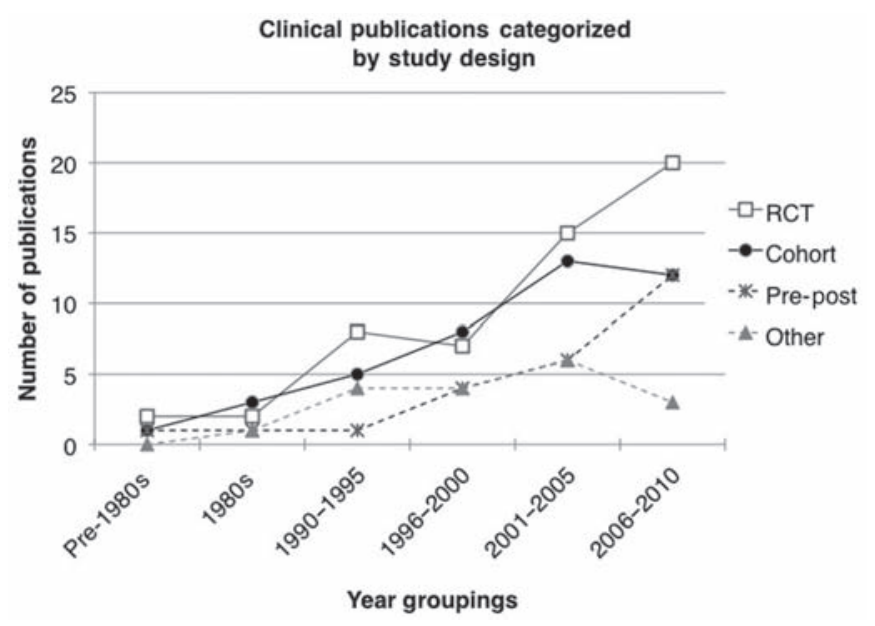

Figure 2 The number of published studies examining injury prevention programmes since 1962 grouped into 5-year intervals and stratified by study design. Articles were categorised as crossover (not shown because $n=0$ ), randomised controlled trials (RCTs), cohort, pre-post (measured before and after intervention) and other (cross-sectional, case-control, case-series, ecological). included a strength and power component, $22.5 \%$ included a stretching component and $16.3 \%$ included some other training protocol.

\section{Sport, injury location and injury type}

Table 2 illustrates the number of articles that studied each individual sport. Soccer was the most frequently studied sport $(n=25)$, followed by football $(n=20)$ and rugby $(n=15)$.

Overall, of the 112 articles that mentioned injury location and sport (figure 5), 68.8\% reported on injuries to the lower extremity and of those, the majority tried to reduce injuries of the type joint (non-bone)-ligament. There were 14 studies that examined interventions specifically designed to reduce concussions, and an additional 10 studies that calculated the incidence of concussion related to injury prevention programme.

Figure 5 provides additional details about sport-specific intervention articles (categorised according to table 2) with respect to injury location and injury type. Of the 118 articles that specifically mentioned particular sports being studied, $41.5 \%$ concerned contact sports whereas $39.8 \%$ and $20.3 \%$ concerned collision and non-contact sports, respectively (the total is greater than $100 \%$ because one article included all three categories). Of the articles that targeted the lower extremities $(\mathrm{n}=50), 56 \%$ were designed for contact sport athletes.

\section{DISCUSSION}

Of the 139 injury prevention articles investigating clinical outcomes, the majority of studies was either RCTs or cohort studies, with RCTs being more common in the past 5 years. Most interventions were related to equipment or training, with fewer studies investigating the effects of changes to rules and regulations. More specifically, most equipment studies examined the effect of braces or taping or head and face protectors, and most training studies examined balance and coordination with strength and power studies increasing more recently. With respect to articles that documented injury location and sport, the vast majority was related to lower extremity, head or spine injuries and studied collision or contact sports.

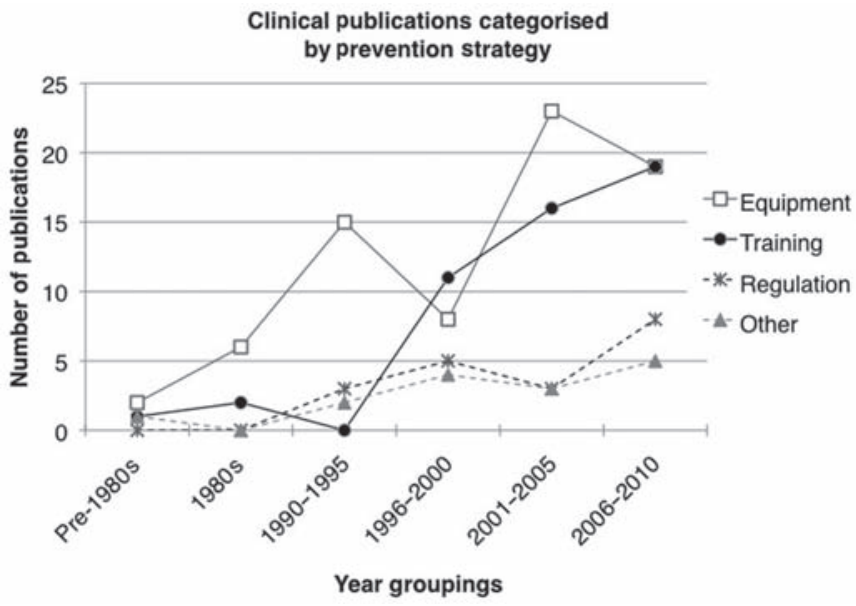

Figure 3 The number of published studies examining injury prevention programmes since 1962 grouped into 5 -year intervals, stratified by intervention type. Equipment refers to interventions using protective devices; training refers to interventions designed to induce a neuromuscular adaptation; regulation refers to changes in rules and regulations; and other is for articles not classified (eg, educational programmes). 
The recent increase in RCTs suggests that the discipline of sport medicine is adopting recommendations by evidencebased medicine organisations. ${ }^{13}$ However, like other areas of medicine, the continued use of cohort studies remains important. When appropriately conducted and interpreted, injury surveillance programmes can provide important insights into the effects of 'natural experiments'. For example, the dramatic

A

Publications categorized by equipment type

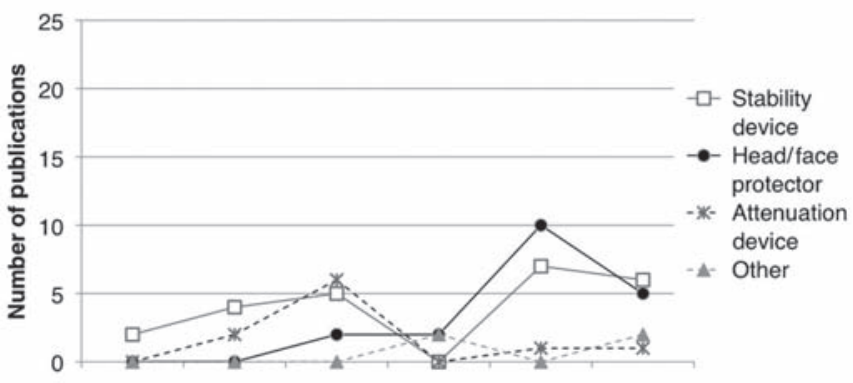

B

Publications categorized by training type

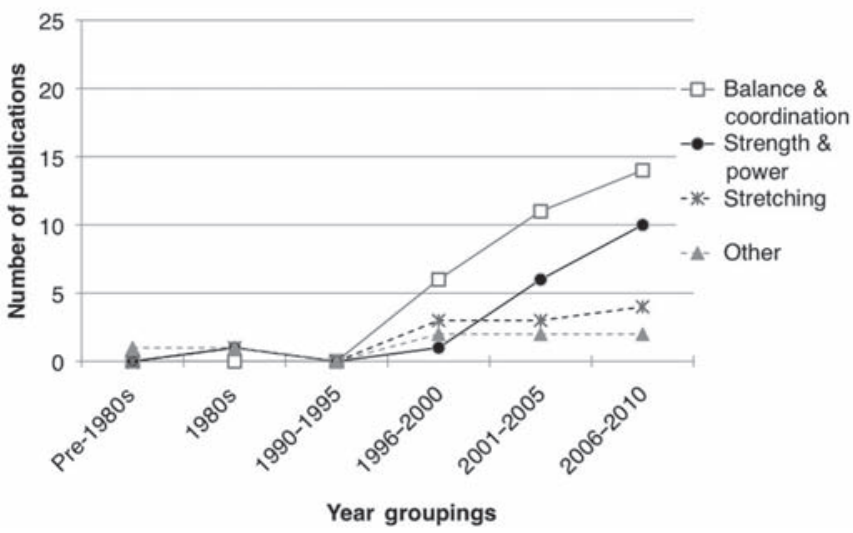

Figure 4 The number of published studies examining injury prevention programmes since 1962 grouped into 5-year intervals, related to equipment interventions $(A)$ and training interventions (B). Equipment interventions included stability devices (eg, brace, tape, orthosis), head and face protectors (eg, helmet, facemask, mouth guard), attenuation devices (eg, shock absorbing insoles) and other (playing surface, thigh protectors). Training interventions included balance/coordination (eg, wobble board), strength/power (eg, resistance training), stretching (eg, effects on position sense) and other (eg, supervised training, technique modification). reduction in cervical spine injuries after outlawing spearing in American Football was obtained by observational cohort studies ${ }^{14}$ and confirms that the rules are important to keep in force. Therefore, under conditions where an outcome is rare and a proposed intervention is highly unlikely to increase the risk of injury, the most effective injury prevention strategy may be to implement changes now and follow-up with observational studies that look at clinical outcomes. An additional example of a rule change that was recently instituted is the National Football League and National Collegiate Athletic Association ban on same-day return to play for concussion. We look forward to reading the observational follow-up studies that assess the impact of this change in regulation as well as others. Finally, observational follow-up studies can also serve to generate ideas for new interventions that can later be tested with RCTs if appropriate.

The fact that all types of intervention strategies have increased since 1990 (figure 3) suggests that the trends are due to an increasing recognition that sports injuries are a public health burden. It also implied that most of these interventions are related to equipment and training with very little research on whether changes to rules and regulations alter injury risk. The findings are likely a result of several factors. First, convincing sport leagues to change the rules is not easy, as rules are an integral part of the sport culture. Second, designing and implementing a randomised study will be expensive, difficult and results would only be available several years later. Third, in leagues where the rules are changed based on lobbying by athletes, clinicians and the public, the effect of the rule change can only be studied if injury surveillance programmes are in place to calculate injury rates both pre- and post rule change; few valid injury surveillance programmes are available at this time. Fourth, where injury surveillance programmes are in place, data access is often limited to only a few individuals or groups. If changing the rules and regulations is going to become an integral part of the sport injury prevention programmes as previously discussed (K McBain, I Shrier, R Shultz et al, manuscript submitted), we will need to find solutions to the above challenges.

Figure $4 \mathrm{~A}$ is a breakdown of the equipment studies over time. A noticeable spike occurred between 2001 and 2005 when head/face protectors were frequently studied. Comparing these results with the studies using a known risk factor as the outcome (K McBain, I Shrier, R Shultz et al, manuscript submitted), the pattern is quite different. Whereas there was a steady and large increase in the number of studies examining

Table 2 Number of articles that studied injury prevention programmes for individual sports

\begin{tabular}{|c|c|c|}
\hline Collision sports & Contact sports & Non-contact sports \\
\hline Football $(n=20)$ & Soccer $(n=25)$ & Cycling ( $n=14)$ \\
\hline Rugby $(n=15)$ & Basketball $(n=12)$ & Running $(\mathrm{n}=7)$ \\
\hline Alpine skiing/snowboarding* $(n=8)$ & Volleyball $(n=6)$ & $\begin{array}{l}\text { Swimming, cricket, hurling, } \\
\text { racquet sports ( } n=1 \text { each) }\end{array}$ \\
\hline Hockey $(n=6)$ & Baseball/softball $(n=5)$ & \\
\hline $\begin{array}{l}\text { Horseback riding, martial arts, bandy } \\
\text { ( } n=1 \text { each) }\end{array}$ & Floorball, gymnastics ( $n=1$ for each) & \\
\hline \multicolumn{3}{|c|}{ There were no injury prevention interventions for the following sports } \\
\hline Bobsleigh, boxing, luge, rodeo & $\begin{array}{l}\text { Fencing, field hockey, figure skating, } \\
\text { judo, kayaking, lacrosse, netball, } \\
\text { weightlifting, wrestling }\end{array}$ & $\begin{array}{l}\text { Archery, badminton, curling, } \\
\text { dance sports, golf, racquetball, } \\
\text { rowing, sailing, shooting, squash, } \\
\text { tennis, water polo }\end{array}$ \\
\hline
\end{tabular}

If a study examined more than one sport, it was counted under each sport.

${ }^{*}$ High risk of high-speed fall/collision with the surface of the hill. 
A Publications categorised by sport type and anatomical site B Publications categorised by sport type and injury type

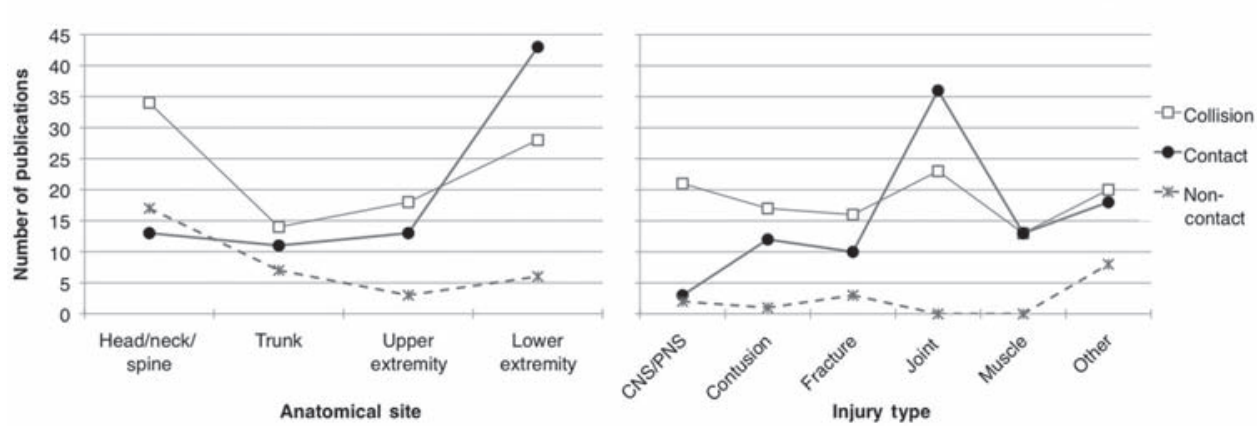

Figure 5 The number of published studies examining injury prevention programmes by injury location $(A)$ and injury type $(B)$ are shown, stratified by the sport studied. We grouped sports according to the risk of contact and the severity of injury expected (table 1).

how stability devices reduce known risk factors for injury from the 1980s to 2000s, only a few studies examine whether these stability devices actually do reduce the number of injuries. These findings likely occur because studies using possible risk factors as the outcome are cheaper and easier to complete. However, the problem is that interventions can sometimes have unexpected consequences. For example, ankle fractures were reduced when low-top alpine ski boots were changed to high-top boots, but the forces involved were redistributed more proximally and are believed to have increased the incidence of tibial fractures and knee injuries. ${ }^{15}$ Although studies using risk factors as outcomes are valuable for certain research questions, researchers and clinicians need to be cognizant that the ultimate objective is to reduce overall injuries.

Figure $4 \mathrm{~B}$ shows that all training intervention strategy studies are increasing. Strength and power studies appear to increase at a similar rate as balance and coordination studies but with a 5 -year delay. The pattern of publications appears similar to that observed in studies that used a known risk factor as the outcome. ${ }^{16}$

Figure 5 shows the number of articles related to injury location, stratified by the type of sport. Collision and contact sports are much more frequently studied than non-contact sports, with a large proportion of studies on collision sports focusing on interventions designed to minimise head/neck/ spine injuries (as expected due to their serious consequences). The large number of non-contact studies examining head/ neck/spine injuries appeared related to a high frequency of studies that assessed the effectiveness of bicycle helmets, and the prevention of overuse injuries to the neck and spine, which remain common. Articles discussing contact and collision sports were generally specific to the lower extremities, which is consistent with the frequency of these injuries in women's varsity basketball and men's football $(-55-60 \%) .{ }^{17}$ 18 The small amount of non-contact and overuse injury research is quite problematic given that these types of injuries represent an important obstacle to regular participation in exercise. ${ }^{19}$

\section{LIMITATIONS}

Although we used a broad search strategy over several electronic databases and scanned the bibliographies of included papers, we may have missed some articles. In order to maximise efficiency, we used a second reviewer to validate all data extraction rather than rely on independent data abstraction. Finally, we limited our study to English language publications and would have missed articles in other languages.

\section{What is already known}

Injury prevention research on clinical outcomes is increasing.

\section{What this study adds}

- An overview of the changing trends in injury prevention research addressing interventions designed to reduce adverse clinical outcomes.

- Identifies gaps in 'clinical outcome' injury prevention research, especially the effects of rule changes on adverse outcomes and interventions to reduce overuse injuries.

There are 139 publications in the English literature examining sports injury interventions. The intervention strategies used in these studies are almost exclusively related to equipment and training in the context of acute injuries in contact or collision sports. There is little intervention research on overuse injuries and almost no research on the effectiveness of rule changes on injury outcomes.

Acknowledgements This publication represents work done by members of RAISEM (Research Alliance in Sport and Exercise Medicine). RAISEM undertakes group research projects on sport injury prevention, clinical treatment and return to play. The members of the group include clinicians, content experts, epidemiologists, biomechanists and physiologists, with associated members collaborating on specific projects.

Competing interests None.

Provenance and peer review Not commissioned; externally peer reviewed.

\section{REFERENCES}

1. Dias RC, Dias JM, Ramos LR. Impact of an exercise and walking protocol on quality of life for elderly people with $\mathrm{OA}$ of the knee. Physiother Res Int 2003;8:121-30.

2. Oka RK, De Marco T, Haskell WL, et al. Impact of a home-based walking and resistance training program on quality of life in patients with heart failure. Am J Cardiol 2000;85:365-9.

3. Blair SN. Effects of physical activity on cardiovascular disease mortality independent of risk factors Physical Activity and Cardiovascular Health: A National Consensus. Champaign, IL: Human Kinetics 1997,:127-36.

4. Siscovick DS, LaPorte RE, Newman JM. The disease-specific benefits and risks of physical activity and exercise. Public Health Rep 1985;100:180-8.

5. Sothern MS, Loftin M, Suskind RM, et al. The health benefits of physical activity in children and adolescents: implications for chronic disease prevention. Eur J Pediatr 1999:158:271-4. 
6. Kra A. Sport-related injuries in an emergency department (prospective study with 310 cases). J Traumato/ Sport 2008;24:204-8.

7. Gabbe BJ, Finch CF, Cameron PA, et al. Incidence of serious injury and death during sport and recreation activities in Victoria, Australia. Br J Sports Med 2005; 39:573-7.

8. Engebretsen L, Bahr R. An ounce of prevention? Br J Sports Med 2005:39:312-13

9. Klügl M, Shrier I, McBain K, et al. The prevention of sport injury: an analysis of 12,000 published manuscripts. Clin J Sport Med 2010;20:407-12.

10. Chalmers DJ, et al. Injury prevention in sport: not yet part of the game? Inj Prev 2002;8(Suppl 4):IV22-5.

11. Shrier I, Meeuwisse WH, Matheson G0, et al. Injury patterns and injury rates in the circus arts: an analysis of 5 years of data from Cirque du Soleil. Am J Sports Med 2009;37:1143-9.

12. Fuller CW, Raftery M, Readhead C, et al. Impact of the International Rugby Board's experimental law variations on the incidence and nature of match injuries in southern hemisphere professional rugby union. S Afr Med J 2009;99:232-7.
13. Bernstein J. Evidence-based medicine. J Am Acad Orthop Surg 2004;12:80-8.

14. Heck JF, Clarke KS, Peterson TR, et al. National Athletic Trainers' Association Position Statement: Head-Down Contact and Spearing in Tackle Football. J Athl Train 2004;39:101-11.

15. Natri A, Beynnon BD, Ettlinger CF, et al. Alpine ski bindings and injuries. Current findings. Sports Med 1999;28:35-48.

16. McBain K, Shrier I, Shultz R, et al. Prevention of Sports Injury I: A Systematic Review of Applied. Br J Sports Med Published Online First: 20 April 2011 doi:10.1136/bjsm.2010.080929.

17. Agel J, Olson DE, Dick R, et al. Descriptive epidemiology of collegiate women's basketball injuries: National Collegiate Athletic Association Injury Surveillance System, 1988-1989 through 2003-2004. J Athl Train 2007:42:202-10.

18. Dick R, Ferrara MS, Agel J, et al. Descriptive epidemiology of collegiate men's football injuries: National Collegiate Athletic Association Injury Surveillance System, 1988-1989 through 2003-2004. J Athl Train 2007;42:221-33.

19. Karlsson J. Risk of overuse injuries-prevention and understanding of individual factors are the key. Scand J Med Sci Sports 2008;18:677-8. 